Pacific

Journal of

Mathematics

THE CLASSIFICATION OF SIMPLY-CONNECTED CONTACT SUB-RIEMANNIAN SYMMETRIC SPACES

Pierre Bieliavsky, Elisha Falbel, and Claudio Gorodski 


\title{
THE CLASSIFICATION OF SIMPLY-CONNECTED CONTACT SUB-RIEMANNIAN SYMMETRIC SPACES
}

\author{
Pierre Bieliavsky, Elisha Falbel, and Claudio Gorodski
}

Sub-Riemannian geometry is the geometry of non-integrable $k$-plane distributions $\mathcal{D}$ on $n$-manifolds $M, 1<k<n$, where $\mathcal{D}$ is equipped with a positive definite metric $g$. We classify the simply-connected contact sub-Riemannian symmetric spaces (these belong to a class of sub-Riemannian manifolds $(M, \mathcal{D}, g)$ with special symmetry properties).

\section{Introduction.}

Sub-Riemannian geometry is the geometry of non-integrable $k$-plane distributions $\mathcal{D}$ on $n$-manifolds $M, 1<k<n$, where $\mathcal{D}$ is equipped with a positive definite metric $g$. See $[\mathbf{2 0}, \mathbf{2 4}, \mathbf{2 5}, \mathbf{2 6}, \mathbf{1 4}, \mathbf{1 9}]$ for an introduction and details on the subject. Note that when $k=n$ we recover Riemannian geometry, but the sub-Riemannian setting includes new interesting phenomena as described in the references above. Sub-Riemannian symmetric spaces constitute a class of sub-Riemannian manifolds $(M, \mathcal{D}, g)$ with special symmetry properties. It is our hope that this class of examples will be valuable in deciphering the features of sub-Riemannian geometry.

This paper completes the classification of simply-connected contact subRiemannian symmetric spaces initiated in $[\mathbf{2 4}, \mathbf{8}, \mathbf{9}, \mathbf{1 0}]$ and provides a link with the symplectic symmetric spaces defined and studied in $[\mathbf{1}, \mathbf{2}]$. This goal is achieved by analysing the involutive Lie algebra naturally attached to the sub-Riemannian symmetric space. It turns out that, in the semisimple case, the sub-Riemannian symmetric space canonically fibers over a base manifold belonging to a subclass of symplectic symmetric spaces. On the other hand, the non-semisimple case includes two cases: The manifold of contact elements of Euclidean space (and its dual) and twisted products of the Heisenberg group with the spaces of the semisimple case. See Table 1 for the full classification.

This work can also be viewed as a first step towards proving a de Rham decomposition theorem for contact sub-Riemannian manifolds. The relation with the holonomy of sub-Riemannian manifolds investigated in [10] will certainly provide the clue for such a result.

Finally, it is worth mentioning here a few other related problems: 
a) The non-simply-connected case, i.e. the problem of studying discrete quotients of contact sub-Riemannian symmetric spaces.

b) The non-contact sub-Riemannian symmetric spaces and their singular geodesics (see [20]).

c) Realizing the underlying CR structure of a sub-Riemannian symmetric space as the boundary of a complex manifold.

The last two authors would like to thank FAPESP and CNPq for partial support during the development of this work. Part of this work was completed when the first and last authors were visiting U. C. Berkeley, for which they thank Prof. J. A. Wolf for his hospitality, and when the second author was visiting U. Paris-Sud, Orsay, for which he thanks Prof. P. Pansu for his hospitality.

\section{Sub-Riemannian symmetric spaces.}

A sub-Riemannian manifold is a triple $(M, \mathcal{D}, g)$ where $M$ is an oriented smooth manifold, $\mathcal{D}$ is an oriented smooth distribution on $M$ and $g$ is a smoothly varying positive definite symmetric bilinear form defined on $\mathcal{D}$.

In this paper we shall consider only the case in which $\mathcal{D}$ is a contact distribution. That means that $\mathcal{D}$ is a codimension one distribution on $M$ and that the Levi form $\mathcal{L}: \mathcal{D} \times \mathcal{D} \rightarrow T M / \mathcal{D}$, defined by $\mathcal{L}(X, Y)=[X, Y]$ $\bmod \mathcal{D}$, is non-degenerate as a skew-symmetric bilinear form on $\mathcal{D}$. Let $\operatorname{dim} M=2 n+1$ and let $d V$ be the volume form on $\mathcal{D}$. The (normalized) contact form is the 1 -form $\theta$ on $M$ such that

$$
\begin{aligned}
\operatorname{ker} \theta & =\mathcal{D}, \\
\left(\left.d \theta\right|_{\mathcal{D}}\right)^{n} & =n ! 2^{n} d V .
\end{aligned}
$$

Since $d \theta$ has maximal rank, there is a unique vector field $\xi$ on $M$ such that

$$
\begin{aligned}
& \theta(\xi)=1, \\
& \iota_{\xi} d \theta=0 .
\end{aligned}
$$

It is called the characteristic vector field. Note that the sub-Riemannian metric $g$ has a natural extension to a Riemannian metric on $M$ by setting $\xi$ to be orthonormal to $\mathcal{D}$.

A local isometry between two sub-Riemannian manifolds $(M, \mathcal{D}, g)$ and $\left(M^{\prime}, \mathcal{D}^{\prime}, g^{\prime}\right)$ is a diffeomorphism between open sets $\psi: U \subset M \rightarrow U^{\prime} \subset M^{\prime}$ such that $\psi_{*}(\mathcal{D})=\mathcal{D}^{\prime}$ and $\psi^{*} g^{\prime}=g$. In the contact case it follows that $\psi^{*} \theta^{\prime}= \pm \theta$ and $\psi_{*} \xi= \pm \xi^{\prime}$ (and therefore $\psi$ will be a local Riemannian isometry relative to the extended Riemannian metrics on $M$ and $M^{\prime}$ ). If $\psi$ is globally defined on $M$ to $M^{\prime}$, we say simply that $\psi$ is an isometry.

A canonical connection analogous to the Levi-Cività connection in the case of Riemannian geometry is uniquely defined on $M$. This connection is 
defined for a contact sub-Riemannian manifold of arbitrary (odd) dimension; in the 3-dimensional case it is the same as the pseudo-Hermitian connection of Webster $([\mathbf{2 7}])$. Let $\underline{T M}$ and $\underline{\mathcal{D}}$ denote respectively the set of sections of $T M$ and of $\mathcal{D}$.

Theorem $1.1([\mathbf{8}, \mathbf{1 1}, \mathbf{1 2}])$. There exists a unique connection $\nabla: \underline{T M} \rightarrow$ $\underline{T M}^{*} \otimes \underline{T M}$, called the adapted connection, and a unique symmetric tensor $\tau: \mathcal{D} \rightarrow \mathcal{D}$, called the sub-torsion, with the following properties ( $T$ is the torsion tensor of the connection):

a. $\nabla_{U}: \underline{\mathcal{D}} \rightarrow \underline{\mathcal{D}}$

b. $\nabla \xi=0$;

c. $\nabla g=0$;

d. $T(X, Y)=d \theta(X, Y) \xi$, $T(\xi, X)=\tau(X)$;

for $X, Y \in \underline{\mathcal{D}}, U \in \underline{T M}$.

Observe that an isometry $\psi: M \rightarrow M^{\prime}$ is affine with respect to the adapted connection, that is, $\nabla_{\psi_{*} X}^{\prime} \psi_{*} Y=\psi_{*}\left(\nabla_{X} Y\right)$ for $X, Y \in \underline{T M}$.

If $(M, \mathcal{D}, g)$ is a sub-Riemannian manifold, it is possible to define a metric space structure on $M$, simply by taking the distance between two points to be the infimum of the $g$-lengths of absolutely continuous curves which are tangent to $\mathcal{D}$ whenever they are differentiable, joining the two points. By Chow's theorem (see one of the surveys cited in the Introduction), the infimum is finite, and defines a bona fide metric distance $d$ on $M$. A subRiemannian geodesic (as opposed to the affine $\nabla$-geodesics) is defined to be a local minimizer with respect to $d$. The contact assumption precludes the appearence of "abnormal" geodesics, so that all geodesics are smooth and, in fact, projections of the trajectories of the Hamiltonian vector field in $T^{*} M$ given by the Legendre transform of the inner product $g$ on $\mathcal{D}$ (see [20]).

In the following, we want to relate three natural notions of completeness for a sub-Riemannian manifold. The following lemma is due to Daniel V. Tausk.

Lemma 1.1. If $M$ is any sub-Riemannian manifold and $\nabla$ is its adapted connection, then any two points in $M$ can be joined by a broken horizontal (i.e. everywhere tangent to $\mathcal{D}) \nabla$-geodesic.

Proof. In fact, given $p, q \in M$, define an equivalence relation $p \sim q$ if and only if they can be joined by such a curve. We check the equivalence classes are open. Fix $p \in M$ and let $q \in M$ be in its equivalence class. It is easy to construct a local horizontal frame field near $q$ such that the integral curves of any vector field in that frame are geodesics. By Chow's theorem, every point sufficiently close to $q$ can be joined to $q$ by a finite sequence of segments of integral curves of vector fields in that frame. It follows that the class of $p$ is open. 
Theorem 1.2. Let $(M, \mathcal{D}, g)$ be a sub-Riemannian manifold. Denote with $\nabla$ the adapted connection and with $\bar{\nabla}$ the Levi-Cività connection associated to the canonical extension of $g$ to a Riemannian metric $\bar{g}$ on $M$. Then the following statements are equivalent:

a. $M$ is $\nabla$-complete;

b. $M$ is $\bar{\nabla}$-complete;

c. $M$ is sR-complete, that is, every sub-Riemannian geodesic in $M$ can be extended indefinitely.

Proof. Let $\bar{d}$ be the metric distance induced by $\bar{g}$ on $M$. Then the identity map $(M, \bar{d}) \rightarrow(M, d)$ is $C^{1 / 2}$-Holder and its inverse is Lipschitz (see [14]). It follows that $(M, \bar{d})$ is a complete metric space if and only if $(M, d)$ is a complete metric space. We apply the Höpf-Rinow theorem and its subRiemannian version (see [24]) to get the equivalence of b. with c.

Now assume b. is true and a. is false and let $\gamma$ be a $\nabla$-geodesic defined on a maximal positive time interval $[0, t)$ with $t<+\infty$. Since $\nabla g=\nabla \bar{g}=0$, we have that $\bar{g}\left(\gamma^{\prime}, \gamma^{\prime}\right)^{1 / 2}$ is constant. Take a sequence $t_{n} \uparrow t$. Then $\left\{\gamma\left(t_{n}\right)\right\}$ is a $\bar{d}$-Cauchy sequence, hence, convergent to a point $q \in M$. If we define $\gamma(t)=q$ then $\gamma$ can be extended beyond $t$, a contradiction.

Finally, we show that a. implies b. and c. Fix $p \in M$. For each integer $n \geq 1$, define $K_{n}$ to be the set of all points in $M$ that can be joined to $p$ by a sequence of at most $n$ horizontal $\nabla$-geodesic segments, each of which of $g$-length at most $n$. Then $\left(K_{n}\right)$ is an increasing sequence of compact subsets of $M$ (because the $\nabla$-exponential map is continuous whichever metric we choose to use in $M, d$ or $\bar{d}$ ) which exhausts $M$ (because of Lemma 1.1).

The definition of sub-symmetric space was given by Strichartz in [24]. Since we have restricted our investigation to contact distributions, we will use a simplified definition. A sub-Riemannian [locally] symmetric space (or sub-symmetric space, for short) is a sub-Riemannian manifold $(M, \mathcal{D}, g)$ such that for every point $x_{0} \in M$ there is an isometry [resp., a local isometry] $\psi$, called the sub-symmetry at $x_{0}$, with $\psi\left(x_{0}\right)=x_{0}$ and $\left.\psi_{*}\right|_{\mathcal{D}_{x_{0}}}=-1$ (in the contact case it follows that $\psi_{*}\left(\xi_{x_{0}}\right)=\xi_{x_{0}}$, where $\xi$ is the characteristic field).

It is easy to see that the sub-symmetry at a point $x_{0}$ must be unique; in fact, it is given by $\exp _{x_{0}}(X) \mapsto \exp _{x_{0}}\left(\psi_{* x_{0}} X\right)$, where exp is the affine exponential map associated to the adapted connection. Observe that the sub-symmetry at $x_{0}$ maps a geodesic passing through $x_{0}$ to itself if and only if the geodesic is horizontal.

Remark 1.1. In $[\mathbf{8}, \mathbf{9}, \mathbf{1 0}]$ we required homogeneity in the definition of sub-symmentric spaces. This in fact follows from the existence of the subsymmetry at all points, as we will see now. 
Theorem 1.3. Let $(M, \mathcal{D}, g)$ be a sub-Riemannian manifold and let $\nabla$ be its adapted connection. Then:

a. $M$ is locally sub-symmetric if and only if $\nabla_{\mathcal{D}} R=\nabla_{\mathcal{D}}{ }^{T}=0$;

b. if $M$ is locally sub-symmetric, then it is locally homogeneous;

c. if $M$ is locally sub-symmetric, $\nabla$-complete and simply-connected, then it is (globally) sub-symmetric;

d. if $M$ is (globally) sub-symmetric, then it is homogeneous.

Proof. a. This was proved in [8].

b. Let $p, p^{\prime} \in M$ and take normal neighborhoods $U=\exp _{p}(V), U^{\prime}=$ $\exp _{p^{\prime}}\left(V^{\prime}\right)$ relative to $\nabla$. Choose any piecewise smooth horizontal curve connecting $p$ and $p^{\prime}$ and let $\phi: T_{p} M \rightarrow T_{p^{\prime}} M$ be the parallel transport along this curve. Since $M$ is locally sub-symmetric, we have $\nabla_{\mathcal{D}} R=\nabla_{\mathcal{D}} T=0$, so $\phi$ sends $R_{p}$ to $R_{p^{\prime}}$ and $T_{p}$ to $T_{p^{\prime}}$. Given $z \in U$, write $z=\exp _{p} v$ for a unique $v \in T_{p} M$ and define $\phi_{z}: T_{z} M \rightarrow T_{z^{\prime}} M, z^{\prime}=\exp _{p^{\prime}} \phi(v)$, to be $\phi_{z}=\tau_{\phi(v)} \phi \tau_{v}^{-1}$, where $\tau_{v}, \tau_{\phi(v)}$ are parallel transport along $t \mapsto \exp _{p} t v$, $t \mapsto \exp _{p^{\prime}} t \phi(v)$, resp. ( $\operatorname{shrink} U$ so that $\left.\exp _{p^{\prime}} \phi(V) \subset U^{\prime}\right)$. Since $\nabla_{\mathcal{D}} R=$ $\nabla_{\mathcal{D}} T=0, R$ and $T$ satisfy a system of ODE's along geodesic rays starting from $p, p^{\prime}$ which have unique solutions for given initial conditions (see [8]). Therefore $\phi_{z}$ sends $R_{z}$ to $R_{z^{\prime}}$ and $T_{z}$ to $T_{z^{\prime}}$. By Cartan's result (see [7], p. 238, or [28]), $f=\exp _{p^{\prime}} \phi \exp _{p}^{-1}: U \rightarrow U^{\prime}$ is an affine diffeomorphism, it is the unique one that induces $\phi$ on $T_{p} M$, and $f_{* z}=\phi_{z}$ for $z \in U$. Hence $f$ is a local (sub-Riemannian) isometry at $p$ with $f(p)=p^{\prime}$ (see Theorem 1.7.18 in $[28])$.

c. Let $p \in M$ and consider the sub-symmetry $\psi: U \rightarrow U$ at $p$. We must show that $\psi$ is globally defined. Recall $\psi(p)=p$ and $\left.\psi_{* p}\right|_{\mathcal{D}_{p}}=-1$. Given a finite sequence $V=\left\{v_{1}, \ldots, v_{r}\right\} \subset T_{p} M$, let $\gamma_{V}$ denote the corresponding broken geodesic in $M$ obtained by following $v_{1}$ for time 1 , then following (the parallel transport to $\exp _{p}\left(v_{1}\right)$ of $) v_{2}$ for time 1, etc., and let $\tau_{V}$ be parallel transport along $\gamma_{V}$ from $p$ to $\gamma_{V}(r)$. Let $\phi_{V}=\tau_{\psi_{*} V} \psi_{* p} \tau_{V}^{-1}$. We have that $\psi_{{ }_{*} p}$ sends $R_{p}$ to $R_{p}$ and $T_{p}$ to $T_{p}$, and since $\nabla_{\mathcal{D}} R=\nabla_{\mathcal{D}} T=0, R$ and $T$ must satisfy a system of ODE's along geodesic rays which has unique solutions for given initial conditions. Hence, $\phi_{V}$ sends $R_{\gamma_{V}(r)}$ to $R_{\gamma_{\psi * V}}(r)$ and $T_{\gamma_{V}(r)}$ to $T_{\gamma_{\psi * V}}(r)$. Therefore $f: M \rightarrow M$ defined by $f\left(\gamma_{V}(r)\right)=\gamma_{\psi_{*} V}(r)$ is a well-defined affine diffeomorphism, it is the unique one which induces $\psi_{*}$ on $T_{p} M$ and the $\phi_{V}$ are the tangent maps of $f$. Clearly, $f$ is an extension of $\psi$ (see Theorem 1.9.1 in [28]).

d. If $\gamma(t)=\exp _{p} t v$ for $p \in M, v \in T_{p} M$, is a horizontal geodesic, i.e. $v \in \mathcal{D}_{p}$, then the sub-symmetry at $\gamma(r / 2)$ interchanges $\gamma(0)$ and $\gamma(r)$. Therefore, it is enough to show that any two points in $M$ can be joined by a broken horizontal geodesic. But this is the contents of Lemma 1.1. 


\section{Involutive Lie algebras.}

An involutive Lie algebra (IL-algebra, for short) is a pair $(\mathfrak{g}, \sigma)$ where $\mathfrak{g}$ is a (real) Lie algebra and $\sigma$ is an involutive automorphism of $\mathfrak{g}$. Then there is a canonical decomposition $\mathfrak{g}=\mathfrak{h}+\mathfrak{p}$ into the \pm 1 -eigenspaces of $\sigma$. We will always assume that $[\mathfrak{p}, \mathfrak{p}]=\mathfrak{h}$.

An orthogonal IL-algebra (OIL-algebra, for short) is a triple $(\mathfrak{g}, \sigma, B)$ where $(\mathfrak{g}, \sigma)$ is an IL-algebra such that $\mathfrak{h}$ is effective on $\mathfrak{p}$ and $B$ is an $\operatorname{ad}_{\mathfrak{h}}$-invariant inner product on $\mathfrak{p}$.

A contact IL-algebra is a triple $(\mathfrak{g}, \sigma, \mathfrak{k})$ where $(\mathfrak{g}, \sigma)$ is an IL-algebra, $\mathfrak{k}$ is a codimension one compact subalgebra of $\mathfrak{h}$ which has an effective action on $\mathfrak{p}$, and the skew-symmetric bilinear form $\Omega: \mathfrak{p} \times \mathfrak{p} \rightarrow \mathfrak{h} / \mathfrak{k}$ defined by $\Omega(X, Y)=[X, Y] \bmod \mathfrak{k}$ is non-degenerate.

A sub-orthogonal IL-algebra (sub-OIL algebra, for short) is a quadruple $(\mathfrak{g}, \sigma, \mathfrak{k}, B)$ where $(\mathfrak{g}, \sigma, \mathfrak{k})$ is a contact IL-algebra and $B$ is an $\operatorname{ad}_{\mathfrak{k}}$-invariant inner product on $\mathfrak{p}$.

A symplectic IL-algebra is a triple $(\mathfrak{g}, \sigma, \Omega)$ where $(\mathfrak{g}, \sigma)$ is an IL-algebra such that $\mathfrak{h}$ is effective on $\mathfrak{p}$ and $\Omega$ is an $\operatorname{ad}_{\mathfrak{h}}$-invariant, non-degenerate skewsymmetric bilinear form on $\mathfrak{p}$ (remark that in this case, the extension of $\Omega$ to $\mathfrak{g}$ by 0 on $\mathfrak{h}$ is a Chevalley 2-cocycle for the trivial representation of $\mathfrak{g}$ on $\mathbb{R})$.

A sub-symplectic IL-algebra is a symplectic IL-algebra $(\mathfrak{g}, \sigma, \Omega)$ such that $\Omega=d \theta$ for some $\theta \in \mathfrak{g}^{*}$ and $\operatorname{ker} \theta \cap \mathfrak{h}$ is a compact subalgebra (we denote the Chevalley coboundary by $d$ ).

An OIL-algebra is the linear object naturally associated to a Riemannian symmetric space, see for instance $[\mathbf{2 8}, \mathbf{1 7}, \mathbf{1 5}]$. In much the same way, a sub-OIL algebra is the linearization of the sub-Riemannian symmetric space structure (see $[\mathbf{8}, \mathbf{9}, \mathbf{1 0}]$ ) and a symplectic IL-algebra is the linearization of the symplectic symmetric space structure (see $[\mathbf{1}, \mathbf{2}, \mathbf{4}]$ ). Next we recall some facts about sub-OIL algebras and later we will explain the relation between contact IL-algebras and sub-symplectic IL-algebras.

Lemma $2.1([8])$. Let $(\mathfrak{g}, \sigma, \mathfrak{k})$ be a contact IL-algebra. Then $\mathfrak{k}$ is an ideal of $\mathfrak{h}$ and we can write $\mathfrak{h}=\mathfrak{k}+\langle\xi\rangle$ where $\xi$ is in the center of $\mathfrak{h}$. Moreover, the restriction of the Killling form $\beta$ of $\mathfrak{g}$ to $\mathfrak{k}$ is negative definite.

Let $(\mathfrak{g}, \sigma, \mathfrak{k}, B)$ be a sub-OIL algebra and consider $\theta \in \mathfrak{g}^{*}$ such that $\theta(\mathfrak{k}+$ $\mathfrak{p})=0$ and $\theta(\xi)=1$. Then $d \theta$ is non-degenerate on $\mathfrak{p}$ and $\theta$ (and $\xi$ ) can be normalized, up to a sign, so that $\left(\left.d \theta\right|_{\mathfrak{p} \times \mathfrak{p}}\right)^{n}$ is a volume form on $\mathfrak{p}$ (the ambiguity in the sign can be fixed by choosing orientations for $\mathfrak{g} / \mathfrak{k}$ and $\mathfrak{p}$ ). Now consider the operator $-\operatorname{ad}_{\xi}: \mathfrak{p} \rightarrow \mathfrak{p}$. Its symmetric part is called the sub-torsion $\tau: \mathfrak{p} \rightarrow \mathfrak{p}$. We say that the sub-OIL algebra is subtorsionless if $\tau=0$. Note that, in this case, $B$ is $\operatorname{ad}_{\mathfrak{h}}$-invariant. More generally, we have 
the formula

$$
-2 B(\tau(X), Y)=B([\xi, X], Y)+B(X,[\xi, Y]),
$$

for $X, Y \in \mathfrak{p}$.

Proposition $2.1([\mathbf{8}])$. Let $(\mathfrak{g}, \sigma, B)$ be a simple Hermitean OIL-algebra. Then $(\mathfrak{g}, \sigma,[\mathfrak{h}, \mathfrak{h}], B)$ is a subtorsionless sub-OIL algebra.

\section{The classification of sub-OIL algebras.}

If $(\mathfrak{g}, \sigma, \mathfrak{k}, B)$ is a sub OIL-algebra, we write $\mathfrak{g}=\mathfrak{h}+\mathfrak{p}$ under $\sigma, \mathfrak{h}=\mathfrak{k}+\langle\xi\rangle$ with $[\mathfrak{k}, \xi]=0$ and $\xi$ normalized by $B$ (see observation after Lemma 2.1) and set $\operatorname{dim} \mathfrak{p}=2 n$. Denote with $\beta$ the Killing form of $\mathfrak{g}$ and with $\Omega: \mathfrak{p} \times \mathfrak{p} \rightarrow \mathfrak{h} / \mathfrak{k}$ the canonical symplectic form. We also have that $[\mathfrak{p}, \mathfrak{p}]=\mathfrak{h}$.

\subsection{Semisimple case.}

Throughout this section we assume that $\mathfrak{g}$ is a semisimple Lie algebra. The classification in the simple case is contained in $[\mathbf{9}, \mathbf{8}, \mathbf{1 0}]$ :

Theorem $3.1([\mathbf{9}, \mathbf{8}, \mathbf{1 0}])$. Let $(\mathfrak{g}, \sigma, \mathfrak{k}, B)$ be a simple sub-OIL algebra. Then, either $\operatorname{ad}_{\mathfrak{h}}$ is irreducible on $\mathfrak{p}$ and $(\mathfrak{g}, \sigma, \mathfrak{k})$ is the underlying contact IL-algebra of the sub-OIL algebra canonically associated to a simple Hermitean OIL-algebra (see Proposition 2.1) (recall the six compact and six non-compact families of simple Hermitean OIL-algebras; here we list the pairs $(\mathfrak{g}, \mathfrak{h})$ :

$$
\begin{array}{ll}
(\mathfrak{s u}(p+q), \mathfrak{s}(\mathfrak{u}(p)+\mathfrak{u}(q))) & (\mathfrak{s u}(p, q), \mathfrak{s}(\mathfrak{u}(p)+\mathfrak{u}(q))) \\
(\mathfrak{s p}(n), \mathfrak{u}(n)) & (\mathfrak{s p}(n, \mathbb{R}), \mathfrak{u}(n)) \\
(\mathfrak{s o}(2 n), \mathfrak{u}(n)) & (\mathfrak{s o}(2 n), \mathfrak{u}(n)) \\
\left(\mathfrak{e}_{6}(-78), \mathfrak{s o}(10)+\mathfrak{s o}(2)\right) & \left(\mathfrak{e}_{6}(-14), \mathfrak{s o}(10)+\mathfrak{s o}(2)\right) \\
\left(\mathfrak{e}_{7}(-133), \mathfrak{e}_{6}+\mathfrak{s o}(2)\right) & \left(\mathfrak{e}_{7}(-25), \mathfrak{e}_{6}+\mathfrak{s o}(2)\right) \\
(\mathfrak{s o}(n+2), \mathfrak{s o}(n)+\mathfrak{s o}(2)), \quad n \neq 2 & (\mathfrak{s o}(n, 2), \mathfrak{s o}(n)+\mathfrak{s o}(2)), \quad n \neq 2)
\end{array}
$$

or $\operatorname{ad}_{\mathfrak{h}}$ is not irreducible on $\mathfrak{p}$ and $(\mathfrak{g}, \mathfrak{h})=(\mathfrak{s o}(1, n+1), \mathfrak{s o}(n)+\mathbb{R})$. In all but the cases $\mathfrak{g}=\mathfrak{s o}(n+2), \mathfrak{s o}(1, n+1)$ and $\mathfrak{s o}(n, 2)$, there is only one $\operatorname{ad}_{\mathfrak{k}}$-invariant inner product $B$ on $\mathfrak{p}$, up to homothety, and the corresponding sub-OIL algebra is subtorsionless. In the other three cases there is a two parameter family of $B$ 's. Moreover, the $\mathfrak{s o}(1, n+1)$ case is never subtorsionless.

Proposition 3.1. There exists a canonical bijection between the set of isomorphism classes of semisimple contact IL-algebras and the set of homothety classes of semisimple sub-symplectic IL-algebras.

Proof. Let $(\mathfrak{g}, \sigma, \mathfrak{k})$ be a semisimple contact IL-algebra. Choose an identification $\iota_{1}: \mathfrak{h} / \mathfrak{k} \rightarrow \mathbb{R}$. This gives rise to a symplectic form $\Omega_{1}$ on $\mathfrak{p}$ which is $\operatorname{ad}_{\mathfrak{h}}$-invariant so that $\left(\mathfrak{g}, \sigma, \Omega_{1}\right)$ is a sub-symplectic IL-algebra. Observe 
that there exists a unique element $\xi \in \mathfrak{h}$ such that $d \beta(\xi, \cdot)=\Omega_{1}$ (since $\left.H^{1}(\mathfrak{g})=H^{2}(\mathfrak{g})=0\right)$. Also, $\beta(\xi, \mathfrak{k})=0$. In particular, since $\xi$ does not belong to $\mathfrak{k}$, the restriction of $\beta$ to $\mathfrak{k} \times \mathfrak{k}$ is non-degenerate and $\langle\xi\rangle=\mathfrak{k}^{\perp_{\beta}} \cap \mathfrak{h}$. This shows that the subspace $\langle\xi\rangle$ is independent of the identification $\iota_{1}$ and that another choice $\iota_{2}$ gives rise to a cocycle $\Omega_{2}$ which is proportional to $\Omega_{1}$. The remainder is immediate.

According to the above proposition, we shall always choose the direction of $\xi$ to be the $\beta$-orthogonal complement to $\mathfrak{k}$ in $\mathfrak{h}$.

Denote by $\mathfrak{g} \rightarrow Z^{2}(\mathfrak{g}): X \rightarrow \underline{X}$ the map defined by the formula $\underline{X}(Y, Z)=$ $\beta(X,[Y, Z])$ and denote by $Z^{2}(\mathfrak{g}) \stackrel{\rho}{\rightarrow} \Lambda^{2}(\mathfrak{p})$ the restriction map to $\mathfrak{p} \times \mathfrak{p}$. An element $\xi$ of $\mathfrak{g}$ is said admissible if its centralizer $C_{\mathfrak{g}}(\xi)$ in $\mathfrak{g}$ is equal to $\mathfrak{h}$. Denote by $\operatorname{Adm}(\mathfrak{g}, \sigma)$ the set of admissible elements.

Proposition 3.2. The mapping $\operatorname{Adm}(\mathfrak{g}, \sigma) \rightarrow \Lambda^{2}(\mathfrak{p}): \xi \rightarrow \rho(\underline{\xi})$ defines a bijection between $\operatorname{Adm}(\mathfrak{g}, \sigma)$ and the set of $\operatorname{ad}_{\mathfrak{h}}$-invariant symplectic forms on $\mathfrak{p}$. It follows that if $t=(\mathfrak{g}, \sigma, \Omega)$ is a semisimple symplectic IL-algebra and if $\mathfrak{g}=\oplus_{i=1}^{r} \mathfrak{g}_{i}$ denotes the canonical decomposition of $\mathfrak{g}$ into a direct sum of simple ideals, then:

a. $\sigma\left(\mathfrak{g}_{i}\right)=\mathfrak{g}_{i}$ for all $i$;

b. setting $t_{i}=\left(\mathfrak{g}_{i},\left.\sigma\right|_{\mathfrak{g}_{i}},\left.\Omega\right|_{\mathfrak{p} \cap \mathfrak{g}_{i} \times \mathfrak{p} \cap \mathfrak{g}_{i}}\right)$, one has the decomposition into a direct sum of symplectic IL-algebras: $t=\oplus_{i=1}^{r} t_{i}$.

Proof. Denote by $\Lambda$ the set of $\operatorname{ad}_{\mathfrak{h}}$-invariant 2 -forms on $\mathfrak{p}$, and by $\Lambda_{0}$ the symplectic ones. Using Jacobi's identity, the invariance of $\beta$ and the definition of $\operatorname{Adm}(\mathfrak{g}, \sigma)$, one checks that $\rho(\operatorname{Adm}(\mathfrak{g}, \sigma)) \subset \Lambda$. Since $(\mathfrak{g}, \sigma)$ is semisimple, one has $\{X \in \mathfrak{p} \mid[X, \mathfrak{h}]=0\}=0$ hence $\operatorname{Adm}(\mathfrak{g}, \sigma) \subset Z(\mathfrak{h})$. The invariance of $\beta$, the non degeneracy of its restriction to $\mathfrak{p} \times \mathfrak{p}$ and the definition of $\operatorname{Adm}(\mathfrak{g}, \sigma)$ yield $\rho(\operatorname{Adm}(\mathfrak{g}, \sigma)) \subset \Lambda_{0}$. Since the restriction of $\beta$ to $\mathfrak{h} \times \mathfrak{h}$ is non-degenerate, one checks that the map $\mathfrak{h} \rightarrow \Lambda^{2}(\mathfrak{p}): h \rightarrow \rho(\underline{h})$ is injective; therefore $\operatorname{Adm}(\mathfrak{g}, \sigma)$ injects into $\Lambda_{0}$. Using an argument identical to the one used in the proof of Proposition 3.1, one observes that $\Omega=\rho(\underline{\xi})$ where $\xi \in Z(\mathfrak{h})$; using the non-degeneracy of $\left.\Omega\right|_{\mathfrak{p} \times \mathfrak{p}}$ one gets $C_{\mathfrak{g}}(\xi)=\mathfrak{h}$ i.e. the map is onto $\Lambda_{0}$. Finally, assume $\sigma\left(\mathfrak{g}_{i}\right) \neq \mathfrak{g}_{i}$ for some $i$. Define $\hat{\mathfrak{g}}=\mathfrak{g}_{i} \oplus \sigma\left(\mathfrak{g}_{i}\right)($ see $[\mathbf{1 7}]), \hat{\sigma}=\left.\sigma\right|_{\hat{\mathfrak{g}}}, \hat{\mathfrak{p}}=\mathfrak{p} \cap \hat{\mathfrak{g}}$ and $\overline{\mathfrak{p}}=\mathfrak{p} \cap \hat{\mathfrak{g}}^{\perp_{\beta}}$. Since $[\hat{\mathfrak{p}}, \overline{\mathfrak{p}}]=0$, $\left.\rho(\underline{\xi})\right|_{\hat{\mathfrak{p}} \times \hat{\mathfrak{p}}}=\left.\Omega\right|_{\hat{\mathfrak{p}} \times \hat{\mathfrak{p}}}$ is non-degenerate; but $\operatorname{Adm}(\hat{\mathfrak{g}}, \hat{\sigma}) \subset Z(\mathfrak{h}) \cap \hat{\mathfrak{g}}=0$, a contradiction.

As a corollary of the proof, one has:

Corollary 3.1. Let $(\mathfrak{g}, \sigma, \Omega)$ be a semisimple symplectic IL-algebra. Then

$$
\operatorname{Adm}(\mathfrak{g}, \sigma)=Z(\mathfrak{h}) \backslash \cup_{i=1}^{r} Z\left(\mathfrak{h}_{i}\right)^{\perp_{\beta}},
$$

where $\mathfrak{h}_{i}=\mathfrak{g}_{i} \cap \mathfrak{h}$. 
Remark 3.1. a. Corollary 3.1 tells us that when $(\mathfrak{g}, \sigma, \Omega)$ is a semisimple symplectic IL-algebra, $\operatorname{Adm}(\mathfrak{g}, \sigma)$ is an open subset of $Z(\mathfrak{h})$ whose connected components are described as follows. For all $i$, fix an element $\xi_{i} \in Z\left(\mathfrak{h}_{i}\right) \backslash\{0\}$. Choose a subset $E \subset\{1, \ldots, r\}$ and define $\Gamma_{E}=\{X \in$ $Z(\mathfrak{h}) \mid \beta\left(X, \xi_{j}\right)>0$ if $j \in E$ and $\beta\left(X, \xi_{l}\right)<0$ if $\left.l \in\{1, \ldots, r\} \backslash E\right\}$. Then, clearly, $\Gamma_{E}$ is a connected component of $\operatorname{Adm}(\mathfrak{g}, \sigma)$ and every connected component is obtained this way; in particular there are $2^{r}$ such connected components.

b. One can show that, if $\mathfrak{g}_{i}$ is absolutely simple (i.e. $\mathfrak{g}_{i}^{c}$ is simple), one has $\operatorname{dim} Z\left(\mathfrak{h}_{i}\right)=1($ see $[\mathbf{1 8}, \mathbf{3}])$.

A symplectic IL-algebra $t=(\mathfrak{g}, \sigma, \Omega)$ is said to be of Hermitean type if there exists a $\Omega$-compatible $\operatorname{ad}_{\mathfrak{h}}$-invariant complex structure $J$ on $\mathfrak{p}$ such that the symmetric bilinear form $B_{J}(X, Y)=\Omega(J X, Y)$ on $\mathfrak{p}$ is positive definite (in particular, $\mathfrak{h}$ must be a compact Lie algebra). An IL-algebra $(\mathfrak{g}, \sigma)$ is said to be of Hermitean type if it is the underlying IL-algebra of a symplectic IL-algebra of Hermitean type. The IL-algebras of Hermitean type are the IL-algebras associated to the Hermitean Riemannian symmetric spaces $([\mathbf{2 8}, \mathbf{1 5}])$. These Hermitean IL-algebras are classified in terms of root systems by the Borel-de Siebenthal-Murakami theorem ([6, 22]); indeed, they are direct sums of simple IL-algebras $(\mathfrak{g}, \sigma)$ where, either $\sigma$ is a Cartan involution of the non-compact $\mathfrak{g}$ such that the associated maximal compact subalgebra admits a non-trivial center, or $(\mathfrak{g}, \sigma)$ is the compact dual to such an algebra; these simple IL-algebras are the six pairs listed in Theorem 3.1.

Lemma 3.1. Let $t=(\mathfrak{g}, \sigma, \Omega)$ be a semisimple symplectic IL-algebra of the Hermitean type. Define $t_{-}=(\mathfrak{g}, \sigma,-\Omega)$. Then, $t$ and $t_{-}$are isomorphic symplectic IL-algebras.

Proof. It is sufficient to prove the lemma for $\mathfrak{g}$ simple (cf. Proposition 3.2) and non-compact (use the duality "compact/non-compact" for irreducible Hermitean symmetric spaces). In this case, $\sigma$ is a Cartan involution of $\mathfrak{g}$ and there exists a Cartan subalgebra $\mathfrak{t}$ of $\mathfrak{g}$ contained in $\mathfrak{h}$. Let $\mathfrak{g}_{u}=\mathfrak{h} \oplus i \mathfrak{p}$ the compact real form of the complexified Lie algebra $\mathfrak{g}^{c}$ obtained from $\sigma$ and denote by $\mathfrak{t}^{c}$ the complexified Cartan subalgebra. One knows that $\operatorname{dim}(Z(\mathfrak{h}))=1$ and that $\Omega=d \xi$ where $\xi \in Z(\mathfrak{h})$. We may assume that $\mathfrak{t}$ was chosen so that $\xi \in \mathfrak{t}$. In order to prove the lemma, it is sufficient to exhibit an automophism $\varphi$ of $\mathfrak{g}$ such that $\varphi(\xi)=-\xi$. One knows that the "rotation" $\rho=-1$ of $\mathfrak{t}^{c}$ extends to an automorphism $\theta_{\rho}$ of $\mathfrak{g}^{c}$ which leaves $\mathfrak{g}_{u}$ invariant (see [28], (8.9.11), p. 267 or $[\mathbf{1 3}, \mathbf{3}]$ ). Therefore $\theta_{\rho}$ leaves $\mathfrak{h}$ invariant (because $\mathfrak{h}$ is the centralizer of $\xi$ in $\mathfrak{g}_{u}$ ); by orthogonality with respect to the Killing form, $\mathfrak{p}_{u}=i \mathfrak{p}$ is $\theta_{\rho}$-invariant, too. The restriction of $\theta_{\rho}$ to $\mathfrak{g}$ provides the desired element $\varphi$. 
Lemma 3.2. Let $t=(\mathfrak{g}, \sigma, \Omega)$ be a semisimple sub-symplectic IL-algebra. If $\mathfrak{h}$ is non-compact, then $\mathfrak{g}$ is simple (and therefore $\mathfrak{g}=\mathfrak{s o}(1, n+1)$, cf. Theorem 3.1).

Proof. Let $t=\oplus_{i=1}^{r} t_{i}$ be the decomposition into simple factors. One first observes that each triple $t_{i}=\left(\mathfrak{g}_{i}, \sigma_{i}, \Omega_{i}\right)$ is a sub-symplectic IL-algebra. For all $i$, one has $\Omega_{i}=d \beta\left(\xi_{i}, \cdot\right)$ with $\xi_{i} \in Z\left(\mathfrak{h}_{i}\right)$ and the $\beta$-orthogonal decomposition $\mathfrak{h}_{i}=\left\langle\xi_{i}\right\rangle \oplus \mathfrak{k}_{i}$, where $\mathfrak{k}_{i}$ is a compact subalgebra of $\mathfrak{h}$ as $t_{i}$ is sub-symplectic; also, $\left[\xi_{i}, \xi_{j}\right]=0$ for all $i, j$. Set $I=\{j \in\{1, \ldots, r\}$ : $\mathfrak{h}_{i}$ is not compact $\}$. Then $\tilde{\mathfrak{k}}=\bigoplus_{j \in I} \mathfrak{k}_{j} \oplus \bigoplus_{l \in\{1, \ldots, r\} \backslash I} \mathfrak{h}_{l}$ is a maximal compact subalgebra of $\mathfrak{h}$. Indeed, let $\overline{\mathfrak{k}}$ be a compact subalgebra of $\mathfrak{h}$ containing $\tilde{\mathfrak{k}}$. The vector space $V=\bigoplus_{j \in I}\left\langle\xi_{j}\right\rangle$ is clearly an Abelian subalgebra of $\mathfrak{h}$ which has a non-compact action on $\mathfrak{p}$ and such that $\mathfrak{h}=\tilde{\mathfrak{k}} \oplus V$. Now, choose $\bar{k} \in \overline{\mathfrak{k}}$ and write $\bar{k}=\tilde{k}+v$ under the above decompostion. The element $\bar{k}-\tilde{k}$ belongs to $\overline{\mathfrak{k}} ;$ in particular $v$ is compact, hence null. So $\tilde{\mathfrak{k}}$ is maximal compact and is therefore conjugated to $\mathfrak{k}$ under an inner automorphism of $\mathfrak{h}$ (see $[\mathbf{2 1}])$. But $\operatorname{cod}_{\mathfrak{h}} \tilde{\mathfrak{k}}=\operatorname{dim} V=\sharp I$; since $\operatorname{cod}_{\mathfrak{h}} \mathfrak{k}=1$, one can suppose $\mathfrak{h}_{i}$ to be compact for all $i \geq 2$ and $\mathfrak{h}_{1}$ non-compact. Since $\operatorname{Inn}(\mathfrak{h}) \subset \operatorname{Aut}(\mathrm{t})$, one can also suppose $\mathfrak{k}=\tilde{\mathfrak{k}}=\left[\mathfrak{h}_{1}, \mathfrak{h}_{1}\right] \bigoplus \oplus_{i=2}^{r} \mathfrak{h}_{i}$. Now, by non degeneracy of $\Omega$, we have $\mathfrak{g}=\mathfrak{g}_{1}$.

Let $(\mathfrak{g}, \sigma)$ be a semisimple IL-algebra of Hermitean type. We denote by $\pi: Z(\mathfrak{h}) \rightarrow P(Z(\mathfrak{h}))$ the projectivization map onto the projective space $P(Z(\mathfrak{h}))$. If $(\mathfrak{g}, \sigma, \mathfrak{k})$ is a contact IL-algebra, we say that $\mathfrak{k}$ determines a contact structure on $(\mathfrak{g}, \sigma)$. Two contact structures on $(\mathfrak{g}, \sigma)$ are equivalent if the associated contact IL-algebras are isomorphic.

Theorem 3.2. $\quad$ a. Under the bijection described in Proposition 3.2, the set of isomorphism classes of contact semisimple non-simple IL-algebras corresponds to the set of homothety classes of semisimple nonsimple sub-symplectic IL-algebras of Hermitean type.

b. Let $(\mathfrak{g}, \sigma)$ be a semisimple IL-algebra of Hermitean type. Choose a connected component $\Gamma$ of $\operatorname{Adm}(\mathfrak{g}, \sigma)$. Then the set of equivalence classes of contact structures on $(\mathfrak{g}, \sigma)$ is parametrized by $\pi(\Gamma)$.

Proof. Item a. follows from Lemma 3.2 and from the fact that if $t=(\mathfrak{g}, \sigma, \Omega)$ is a semisimple symplectic IL-algebra such that $\mathfrak{h}$ is compact then $t$ is of Hermitean type. In order to prove this fact, one can assume that $\mathfrak{g}$ is simple and non-compact. In this case $\sigma$ is a Cartan involution and $Z(\mathfrak{h})$ is onedimensional. For all $Z \in Z(\mathfrak{h}) \backslash\{0\}$, one has $\operatorname{ker}(\operatorname{ad} z \mid \mathfrak{p})=0$; indeed, $\mathfrak{g}$ being simple, Corollary 3.1 tell us that $\operatorname{Adm}(\mathfrak{g}, \sigma)=Z(\mathfrak{h}) \backslash\{0\}$. Therefore, $\operatorname{ker}\left(\left.\operatorname{ad}_{\mathbf{Z}}\right|_{\mathfrak{p}}\right) \neq 0$ would contradict the non degeneracy of $\rho(\underline{Z})$. Now, by compactness, one gets an element $Z_{0} \in Z(\mathfrak{h}) \backslash\{0\}$ such that $J=\operatorname{ad}_{\mathrm{Z}_{0}} \mid \mathfrak{p}$ 
defines the desired complex structure. Item b. follows from Propositions 3.1 and 3.2, Lemma 3.1 and the fact that $\mathfrak{h}$ is compact.

It remains to analyse the sub-Riemannian metrics.

Theorem 3.3. Let $(\mathfrak{g}, \sigma, \mathfrak{k}, B)$ be a semisimple non-simple sub-OIL algebra. If $\operatorname{dim} \mathfrak{p}>4$ then the subtorsion $\tau$ vanishes. In particular, $(\mathfrak{g}, \sigma, B)$ is a Hermitean OIL-algebra and the set of equivalence classes of contact structures on $(\mathfrak{g}, \sigma)$ is described in Theorem 3.2.

Proof. Let $\xi$ be an element of $Z(\mathfrak{h})$ associated to $\mathfrak{k}$. According to Corollary 3.1, one has $\xi=\sum_{i=1}^{r} \xi_{i}$ where $\xi_{i} \in Z\left(\mathfrak{h}_{i}\right) \backslash\{0\}$. Defining, for all $k=1, \ldots, r, a_{k}=-\beta\left(\xi^{(k)}, \xi^{(k)}\right) / \beta\left(\xi_{k}, \xi_{k}\right)$ where $\xi^{(k)}=\sum_{i \neq k} \xi_{i}$, one checks that $\eta_{k}=\xi^{(k)}+a_{k} \xi_{k}$ belongs to $\mathfrak{k}$ as $\beta\left(\eta_{k}, \xi\right)=0$. For all $i, j ; i \neq j$, one has $\left.\operatorname{ad}_{\xi}\right|_{\mathfrak{p}_{i}}=\operatorname{ad}_{\eta_{\mathrm{j}}} \mid \mathfrak{p}_{\mathrm{i}}$. This implies, since $r \geq 2$, that $B_{i}=\left.B\right|_{\mathfrak{p}_{i} \times \mathfrak{p}_{i}}$ is $\operatorname{ad}_{\mathfrak{h}^{-}}$ invariant (note that this is true even if $\operatorname{dim} \mathfrak{p}=4$ ). The condition $r \geq 3$ implies that the subtorsion $\tau$ vanishes; indeed, choose $i \neq k \neq j$, then for $X_{i} \in \mathfrak{p}_{i}, X_{j} \in \mathfrak{p}_{j}$ we have

$$
\begin{aligned}
-2 B\left(\tau\left(X_{i}\right), X_{j}\right) & =B\left(\left[\xi, X_{i}\right], X_{j}\right)+B\left(X_{i},\left[\xi, X_{j}\right]\right) \\
& =B\left(\left[\eta_{k}, X_{i}\right], X_{j}\right)+B\left(X_{i},\left[\eta_{k}, X_{j}\right]\right) \\
& =0 .
\end{aligned}
$$

Therefore, $B$ is $\operatorname{ad}_{\mathfrak{h}}$-invariant and the proposition is proved in the case $r \geq 3$. Assume in the following that $r=2$ and $\operatorname{dim} \mathfrak{p}>4$.

Without loss of generality, one can suppose $\operatorname{dim} \mathfrak{p}_{1} \geq 4$. Therefore $\left(\mathfrak{g}_{1}\right.$, $\sigma \mid \mathfrak{g}_{1}$ ) is a simple IL-algebra of Hermitean type such that $\mathfrak{k}_{1} \neq 0$ (indeed, if $\mathfrak{k}_{1}=0$ then $\mathfrak{g}_{1}$ cannot be simple, see [8], Theorem 4.1). Let $V=\left[\mathfrak{k}_{1}, \mathfrak{p}_{1}\right] \subset \mathfrak{p}_{1}$. Since $\xi_{1}$ is central in $\mathfrak{h}_{1}, V$ is $\operatorname{ad}_{\mathfrak{h}_{1}}$-invariant. Then $V=0$ or $V=\mathfrak{p}_{1}$, because $\operatorname{ad}_{\mathfrak{h}_{1}}$ is irreducible on $\mathfrak{p}_{1}$. But $\mathfrak{k}_{1}$ is effective on $\mathfrak{p}_{1}$, which rules out the former possibility. Therefore, $\left[\mathfrak{k}_{1}, \mathfrak{p}_{1}\right]=\mathfrak{p}_{1}$ and one has $B\left(\mathfrak{p}_{1}, \mathfrak{p}_{2}\right)=B\left(\left[\mathfrak{k}_{1}, \mathfrak{p}_{1}\right], \mathfrak{p}_{2}\right)=$ $B\left(\mathfrak{p}_{1},\left[\mathfrak{k}_{1}, \mathfrak{p}_{2}\right]\right)=0$ which implies $B$ is $\operatorname{ad}_{\mathfrak{h}}$-invariant (since $B_{1}$ and $B_{2}$ are already $\operatorname{ad}_{\mathfrak{h}}$-invariant).

Theorem 3.4. Let $(\mathfrak{g}, \sigma, \mathfrak{k})$ be a semisimple non-simple contact IL-algebra such that $\operatorname{dim} \mathfrak{p}=4$. Then, $r=2$ and $\mathfrak{g}_{i}^{c}$ has the type $A_{1}(i=1,2)$; in particular, $\mathfrak{h}=\mathfrak{u}(1) \oplus \mathfrak{u}(1)$. Moreover, every $\operatorname{ad}_{\mathfrak{k}}$-invariant sub-Riemannian structure $B$ has a vanishing associated subtorsion $\tau_{B}$ if and only if $\mathfrak{k}=$ $\left\langle Z_{1} \oplus Z_{2}\right\rangle$ with $Z_{1} \neq Z_{2} ; Z_{1}, Z_{2} \in \mathfrak{u}(1) \backslash\{0\}$ and in this case one has a one-parameter family of sub-Riemannian metrics, up tp homothety. In the case $\mathfrak{k}=\langle Z \oplus Z\rangle ; Z \in \mathfrak{u}(1) \backslash\{0\}$, one has a three-parameter family of $\operatorname{ad}_{\mathfrak{k}}$ - invariant sub-Riemannian structures (one-parameter with vanishing associated subtorsion).

Proof. By direct computation. 


\subsection{Non-semisimple case.}

Throughout this section we assume that $(\mathfrak{g}, \sigma, \mathfrak{k}, B)$ is a non-semisimple sub-OIL algebra.

Theorem 3.5. Let $n \geq 2$. If $\mathfrak{g}$ is a solvable Lie algebra, then $\mathfrak{k}=0$ and $(\mathfrak{g}, \sigma, 0, B)$ is the Heisenberg sub-OIL algebra.

Proof. We have $\mathfrak{k}=[\mathfrak{p}, \mathfrak{p}] \cap \mathfrak{k}, \beta(\mathfrak{g},[\mathfrak{g}, \mathfrak{g}])=0$ and $\beta$ is negative definite on $\mathfrak{k}$. Therefore $\mathfrak{k}=0$. Now Theorem 4.1 in $[\mathbf{8}]$ implies that $\mathfrak{g}$ is the Heisenberg algebra.

Let $\mathfrak{r}$ be the radical of $\mathfrak{g}$. Because of Theorem 3.5, we may assume that $0 \neq \mathfrak{r} \neq \mathfrak{g}$. If $\mathfrak{s} \neq 0$ is an $\operatorname{ad}_{\mathfrak{k}^{-}}, \sigma$ - invariant Levi subalgebra of $\mathfrak{g}$ (cf. [17]), write $\mathfrak{h}_{r}=\mathfrak{h} \cap \mathfrak{r}, \mathfrak{h}_{s}=\mathfrak{h} \cap \mathfrak{s}, \mathfrak{p}_{r}=\mathfrak{p} \cap \mathfrak{r}$ and $\mathfrak{p}_{s}=\mathfrak{p} \cap \mathfrak{s}$. Then $\mathfrak{r}=\mathfrak{h}_{r}+\mathfrak{p}_{r}$ and $\mathfrak{s}=\mathfrak{h}_{s}+\mathfrak{p}_{s}$ and $\mathfrak{g}=\mathfrak{r}+\mathfrak{s}$ semidirect sum of IL-algebras. Note also that $\mathfrak{r} \neq 0$ implies that $\mathfrak{p}_{r} \neq 0$.

Lemma 3.3. We have $\mathfrak{k} \cap\left[\mathfrak{p}, \mathfrak{p}_{r}\right]=0$.

Proof. This follows since $\beta$ is negative definite on $\mathfrak{k}, \beta(\mathfrak{r},[\mathfrak{g}, \mathfrak{g}])=0$ and $\left[\mathfrak{p}, \mathfrak{p}_{r}\right] \subset \mathfrak{r} \cap[\mathfrak{g}, \mathfrak{g}]$.

Lemma 3.4. We have $\left[\mathfrak{p}, \mathfrak{p}_{r}\right]=\mathfrak{h}_{r}$ and $\left[\mathfrak{p}_{s}, \mathfrak{p}_{s}\right]=\mathfrak{h}_{s}$. In particular, $\operatorname{dim} \mathfrak{h}_{r}=$ 1 and we may take $\mathfrak{h}_{r}=\langle\xi\rangle$.

Proof. Use the facts that $\mathfrak{r}$ is an ideal, $\mathfrak{s}$ is a subalgebra and $[\mathfrak{p}, \mathfrak{p}]=\mathfrak{h}$ to conclude that $\left[\mathfrak{p}, \mathfrak{p}_{r}\right]=\mathfrak{h}_{r}$ and $\left[\mathfrak{p}_{s}, \mathfrak{p}_{s}\right]=\mathfrak{h}_{s}$; since $\Omega$ is exact and nondegenerated on $\mathfrak{p}$, this implies $\mathfrak{h}_{r} \neq 0$. Now $\mathfrak{h}_{r} \not \subset \mathfrak{k}$ (Lemma 3.3) and $\left[\mathfrak{k}, \mathfrak{h}_{r}\right] \subset \mathfrak{k} \cap \mathfrak{h}_{r}=0$ (k and $\mathfrak{h}_{r}$ are ideals in $\mathfrak{h}$ ), so $\mathfrak{h}_{r}$ is complementary to $\mathfrak{k}$ in $\mathfrak{h}$ and in the centralizer of $\mathfrak{k}$.

Lemma 3.5. If $\mathfrak{h}_{s} \not \subset \mathfrak{k}$, then $\left[\xi, \mathfrak{p}_{s}\right]=0$.

Proof. Suppose $W=\xi+Z \in \mathfrak{h}_{s}$, with $Z \in \mathfrak{k}$. Then $\left[\xi, \mathfrak{p}_{s}\right] \subset \mathfrak{p}_{s} \cap \mathfrak{p}_{r}=0$.

Lemma 3.6. If $\left[\xi, \mathfrak{p}_{s}\right]=0$, then $\left[\mathfrak{h}_{s}, \mathfrak{p}_{r}\right]=0$.

Proof. We have $\left[\mathfrak{h}_{s}, \mathfrak{p}_{r}\right]=\left[\left[\mathfrak{p}_{s}, \mathfrak{p}_{s}\right], \mathfrak{p}_{r}\right] \subset\left[\mathfrak{p}_{s},\left[\mathfrak{p}_{s}, \mathfrak{p}_{r}\right]\right] \subset\left[\mathfrak{p}_{s}, \xi\right]=0$.

Lemma 3.7. If $\left[\mathfrak{h}_{s}, \mathfrak{p}_{r}\right]=0$, then $[\mathfrak{r}, \mathfrak{s}]=0$.

Proof. We have:

a. $\left[\mathfrak{h}_{r}, \mathfrak{h}_{s}\right]=0$ because $\mathfrak{h}_{r}=\langle\xi\rangle$ is in the center of $\mathfrak{h}$.

b. $\left[\mathfrak{p}_{r}, \mathfrak{p}_{s}\right]=0$ because

$$
\left[\mathfrak{p}_{r}, \mathfrak{p}_{s}\right]=\left[\mathfrak{p}_{r},\left[\mathfrak{h}_{s}, \mathfrak{p}_{s}\right]\right]=\left[\left[\mathfrak{p}_{r}, \mathfrak{h}_{s}\right], \mathfrak{p}_{s}\right]+\left[\left[\mathfrak{p}_{r}, \mathfrak{p}_{s}\right], \mathfrak{h}_{s}\right] \subset\left[\mathfrak{h}_{r}, \mathfrak{h}_{s}\right]=0 .
$$

c. $\left[\mathfrak{h}_{r}, \mathfrak{p}_{s}\right]=0$ because

$$
\left[\mathfrak{h}_{r}, \mathfrak{p}_{s}\right]=\left[\mathfrak{h}_{r},\left[\mathfrak{h}_{s}, \mathfrak{p}_{s}\right]\right]=\left[\left[\mathfrak{h}_{r}, \mathfrak{h}_{s}\right], \mathfrak{p}_{s}\right]+\left[\left[\mathfrak{h}_{r}, \mathfrak{p}_{s}\right], \mathfrak{h}_{s}\right] \subset\left[\mathfrak{p}_{r}, \mathfrak{h}_{s}\right]=0 .
$$




\subsection{1. $\mathfrak{p}_{r}$ is symplectic.}

Throughout this section, we assume that the restriction of $\Omega$ to $\mathfrak{p}_{r}$ is a symplectic form.

Lemma $3.8([\mathbf{1}, \mathbf{2}])$. There exists one and only one $\operatorname{ad}_{\mathfrak{k}}-, \sigma$-invariant Levi subalgebra $\mathfrak{s}$ of $\mathfrak{g}$ such that $\mathfrak{g}$ decomposes as a direct sum of ideals $\mathfrak{r}+\mathfrak{s}$. Moreover, $(\mathfrak{r}, \sigma \mid \mathfrak{r}, 0)$ is the Heisenberg contact IL-algebra and $B\left(\mathfrak{p}_{r}, \mathfrak{p}_{s}\right)=0$.

Proof. Define $\mathfrak{p}_{1}$ to be the symplectic orthogonal of $\mathfrak{p}_{r}$ in $\mathfrak{p}$. Then $\mathfrak{p}=\mathfrak{p}_{r}+\mathfrak{p}_{1}$, $\operatorname{ad}_{\mathfrak{h}}$-invariant decomposition, and $\left[\mathfrak{h}_{r}, \mathfrak{p}_{1}\right] \subset \mathfrak{p}_{1} \cap \mathfrak{p}_{r}=0$. Now define $\mathfrak{h}_{1}=$ $\left[\mathfrak{p}_{1}, \mathfrak{p}_{1}\right]$. We have $\left[\mathfrak{h}_{1}, \mathfrak{p}_{r}\right]=0$ because

$$
\left[\mathfrak{h}_{1}, \mathfrak{p}_{r}\right] \subset\left[\left[\mathfrak{p}_{1}, \mathfrak{p}_{r}\right], \mathfrak{p}_{1}\right] \subset\left[\mathfrak{h}_{r}, \mathfrak{p}_{1}\right]=0 .
$$

Next we show that $\mathfrak{r}$ with the induced structure of contact IL-algebra is the Heisenberg contact IL-algebra. In fact, if $\operatorname{dim} \mathfrak{r} \geq 5$ this follows from Theorem 3.5. If $\operatorname{dim} \mathfrak{r}=3$, this follows from the fact that $\mathfrak{r}$ is subtorsionless with respect to $\left.B\right|_{\mathfrak{p}_{r} \times \mathfrak{p}_{r}}$ : Since $\mathfrak{p}_{1}$ is symplectic, $\mathfrak{h}_{1} \not \subset \mathfrak{k}$ and then there is $W=\xi+Z \in \mathfrak{h}_{1}$ with $Z \in \mathfrak{k} ;$ now $\operatorname{ad}_{\xi}\left|\mathfrak{p}_{r}=-\operatorname{ad}_{Z}\right|_{\mathfrak{p}_{r}}$.

The above considerations imply that $[\xi, \mathfrak{p}]=0$. If $\mathfrak{s}$ is any $\operatorname{ad}_{\mathfrak{k}^{-}}, \sigma$-invariant Levi subalgebra of $\mathfrak{g}$, then Lemmas 3.6 and 3.7 imply that $[\mathfrak{r}, \mathfrak{s}]=0$. Moreover, $\Omega\left(\mathfrak{p}_{s}, \mathfrak{p}_{r}\right)=\Omega\left(\left[\mathfrak{h}_{s}, \mathfrak{p}_{s}\right], \mathfrak{p}_{r}\right)=\Omega\left(\mathfrak{p}_{s},\left[\mathfrak{h}_{s}, \mathfrak{p}_{r}\right]\right)=0$, hence $\mathfrak{p}_{s}=\mathfrak{p}_{1}$ and a similar argument yields that $B\left(\mathfrak{p}_{s}, \mathfrak{p}_{r}\right)=0$.

It follows that:

Theorem 3.6. Let $(\mathfrak{g}, \sigma, \mathfrak{k}, B)$ be a sub-OIL algebra such that $\mathfrak{g}$ is not semisimple nor solvable and $\mathfrak{p}_{r}$ is a $\Omega$-symplectic space. Then $\mathfrak{g}=\mathfrak{r}+\mathfrak{s}$ direct sum of IL-algebras where $\left(\mathfrak{r}, \sigma|\mathfrak{r}, 0, B| \mathfrak{p}_{r} \times \mathfrak{p}_{r}\right)$ is the Heisenberg sub-OIL algebra, $\left(\mathfrak{s},\left.\sigma\right|_{\mathfrak{s}}, \mathfrak{k} \cap \mathfrak{s},\left.B\right|_{\mathfrak{p}_{s} \times \mathfrak{p}_{s}}\right)$ is a subtorsionless, semisimple sub-OIL algebra and $\mathfrak{k}=\mathfrak{k} \cap \mathfrak{s}+\left\langle\xi-\xi_{s}\right\rangle$ where $\left\langle\xi_{s}\right\rangle=(\mathfrak{k} \cap \mathfrak{s})^{\perp \beta} \cap \mathfrak{h}_{s}$ and $\xi$, $\xi_{\text {s }}$ are normalized by $B,\left.B\right|_{\mathfrak{p}_{s} \times \mathfrak{p}_{s}}$. The sub-OIL algebra $(\mathfrak{g}, \sigma, \mathfrak{k}, B)$ is subtorsionless.

\subsection{2. $\mathfrak{p}_{r}$ is not symplectic.}

Throughout this section, we assume that the restriction of $\Omega$ to $\mathfrak{p}_{r}$ is not a symplectic form.

If $\mathfrak{s}$ is any Levi subalgebra of $\mathfrak{g}$, then $[\mathfrak{r}, \mathfrak{s}] \neq 0$. By Lemmas 3.5, 3.6 and 3.7, it follows that $\mathfrak{h}_{s} \subset \mathfrak{k}$. Therefore, $\mathfrak{h}_{s}=\mathfrak{k}$ and $\mathfrak{p}_{s}$ is isotropic (i.e. $\left.\Omega\left(\mathfrak{p}_{s}, \mathfrak{p}_{s}\right)=0\right)$. Also, $\mathfrak{q}=\left\{Y \in \mathfrak{p}_{r}: \Omega\left(Y, \mathfrak{p}_{r}\right)=0\right\} \neq 0$.

Lemma 3.9. We have $\left[\xi, \mathfrak{p}_{r}\right]=0$ and $\left[\mathfrak{p}_{r}, \mathfrak{p}_{r}\right]=0$. Therefore, $\mathfrak{p}_{r}$ and $\mathfrak{p}_{s}$ are Lagrangian (i.e. maximally isotropic) and $\mathfrak{r}$ is Abelian.

Proof. Choose $Y \in \mathfrak{q}$ and $X \in \mathfrak{p}_{s}$ such that $[X, Y]=\xi$ (we have $\left[\mathfrak{q}, \mathfrak{p}_{s}\right] \neq 0$ by definition of $\mathfrak{q}$ and non-degeneracy of $\Omega$ ). By definition of $\mathfrak{q}$, we get $\left[\mathfrak{q}, \mathfrak{p}_{r}\right]=0$ and, since

$$
\Omega([\xi, \mathfrak{q}], \mathfrak{p})=\Omega(\mathfrak{q},[\xi, \mathfrak{p}])=\Omega\left(\mathfrak{q}, \mathfrak{p}_{r}\right)=0,
$$


we have $[\xi, \mathfrak{q}]=0$. Now

$$
\left[\xi, \mathfrak{p}_{r}\right]=\left[\left[\mathfrak{p}_{r}, X\right], Y\right]+\left[\left[Y, \mathfrak{p}_{r}\right], X\right] \subset\left[\left[\mathfrak{p}_{r}, \mathfrak{q}\right], \mathfrak{p}_{s}\right]+[\xi, \mathfrak{q}]=0,
$$

and $\left[\left[\mathfrak{p}_{r}, \mathfrak{p}_{r}\right], \mathfrak{p}\right] \subset\left[\left[\mathfrak{p}_{r}, \mathfrak{p}\right], \mathfrak{p}_{r}\right]=\left[\xi, \mathfrak{p}_{r}\right]=0$. We conclude that $\left[\mathfrak{p}_{r}, \mathfrak{p}_{r}\right]=0$ because $\mathfrak{h}$ is effective on $\mathfrak{p}$ by Lemmas 3.6 and 3.7.

Now $\mathfrak{p}_{r}$ and $\mathfrak{p}_{s}$ are $\operatorname{ad}_{\mathfrak{k}}$-equivariantly isomorphic under $\Omega$. In particular, we have that $\operatorname{dim} \mathfrak{p}_{r}=\operatorname{dim} \mathfrak{p}_{s}=n \geq 2$.

Lemma 3.10. We have $\operatorname{ad}_{\xi}: \mathfrak{p}_{s} \rightarrow \mathfrak{p}_{r}$ is an $\left(\operatorname{ad}_{\mathfrak{k}}\right.$-equivariant) isomorphism.

Proof. In fact, define $\overline{\mathfrak{p}_{s}}$ to be the centralizer of $\xi$ in $\mathfrak{p}_{s}$. Jacobi implies that this is an $\operatorname{ad}_{\mathfrak{h}_{s}}$-invariant subspace; the complete reducibility of $\mathfrak{h}_{s}=\mathfrak{k}$ on $\mathfrak{p}$ and $[\mathfrak{s}, \mathfrak{s}]=\mathfrak{s}$ then yield $\left[\mathfrak{h}_{s}, \overline{\mathfrak{p}_{s}}\right]=\overline{\mathfrak{p}_{s}}$. Therefore

$$
\begin{aligned}
\Omega\left(\left[\mathfrak{h}_{s}, \overline{\mathfrak{p}_{s}}\right], \mathfrak{p}_{r}\right) & =\Omega\left(\overline{\mathfrak{p}_{s}},\left[\mathfrak{h}_{s}, \mathfrak{p}_{r}\right]\right) \\
& =\Omega\left(\overline{\mathfrak{p}_{s}},\left[\mathfrak{p}_{s},\left[\mathfrak{p}_{s}, \mathfrak{p}_{r}\right]\right]\right) \\
& =\Omega\left(\overline{\mathfrak{p}_{s}},\left[\mathfrak{p}_{s}, \xi\right]\right) \\
& =\Omega\left(\left[\xi, \overline{\mathfrak{p}_{s}}\right], \mathfrak{p}_{s}\right) \\
& =0 .
\end{aligned}
$$

Thus, $\overline{\mathfrak{p}_{s}}=\left[\mathfrak{h}_{s}, \overline{\mathfrak{p}_{s}}\right]=0$.

Now $\mathfrak{s}=\mathfrak{k}+\mathfrak{p}_{s}$ is a semisimple OIL-algebra, and the calculation in [10] shows that it is a constant curvature simple OIL-algebra. Thus, $\mathfrak{g}=\mathfrak{s o}(n+$ 1) $\bowtie \mathbb{R}^{n+1}$ or $\mathfrak{g}=\mathfrak{s o}(1, n) \bowtie \mathbb{R}^{n+1}$ and $\mathfrak{k}=\mathfrak{s o}(n)$, as in [10]. Therefore,

Theorem 3.7. Let $(\mathfrak{g}, \sigma, \mathfrak{k}, B)$ be a sub-OIL algebra such that $\mathfrak{g}$ is neither semisimple nor solvable and $\mathfrak{p}_{r}$ is not a $\Omega$-symplectic space. Then the associated sub-Riemannian symmetric space is either the manifold of contact elements of Euclidean space $S O(n+1) \bowtie \mathbb{R}^{n+1} / S O(n)$ or its dual $S O(1, n) \bowtie \mathbb{R}^{n+1} / S O(n)($ see $[\mathbf{1 0}])$.

Theorems 3.1, 3.3, 3.4, 3.5, 3.6 and 3.7 put together complete the classification of simply-connected sub-Riemannian symmetric spaces.

\section{CR manifolds.}

Let $M$ be a smooth manifold equipped with a contact distribution $\mathcal{D}$ and suppose that a complex structure $J$ is defined on $\mathcal{D}$, that is, $J$ is a smooth bundle endomorphism $\mathcal{D} \rightarrow \mathcal{D}$ such that $J_{x}^{2}=-1$ for all $x \in M$. Decompose the complexification $\mathcal{D}^{c}=\mathcal{D}^{1,0} \oplus \mathcal{D}^{0,1}$ into the $\pm \imath$-eigenbundles of $J$. We say that $(M, \mathcal{D}, J)$ is a Cauchy-Riemann manifold (or CR-manifold, for short) if the (real) distribution $\mathcal{D}^{1,0}$ is involutive. It is well known (see [16]) that a sufficient condition for that is that for all $X, Y \in \mathcal{D}$ we have:

$$
J[J X, Y]-J[X, J Y] \in \mathcal{D}
$$


and the Nijenhuis tensor

$$
N(X, Y)=[J X, J Y]-[X, Y]-J[J X, Y]-J[X, J Y]
$$

vanishes.

Now let $(M, \mathcal{D}, g)$ be a sub-Riemannian manifold of contact type, and consider the normalized contact 1 -form $\theta$. It is known that from this data we get a canonical complex structure $J$ on $\mathcal{D}$. In fact, if $H: \mathcal{D} \rightarrow \mathcal{D}$ is defined by

$$
d \theta(X, Y)=g(H X, Y)
$$

for $X, Y \in \mathcal{D}$, we let $J$ be the orthogonal component in the polar decomposition of $H$; see [23]. In this case, condition (1) is automatic, as it follows from the fact that $d \theta(J X, J Y)=d \theta(X, Y)$.

In the particular case when $(M, \mathcal{D}, g)$ is a sub-Riemannian symmetric space, $J$ is clearly invariant under the sub-symmetries. Now condition (2) holds too, because $N$ is a tensor of odd degree which is invariant under the sub-symmetries. In this way, for each space in the classification table we get an example of a homogeneous CR manifold. Finally, we note that for each one of these spaces we have also that $\nabla J=0$ (sub-Kahler condition) as again we have here a tensor of odd degree invariant under the sub-symmetries. 


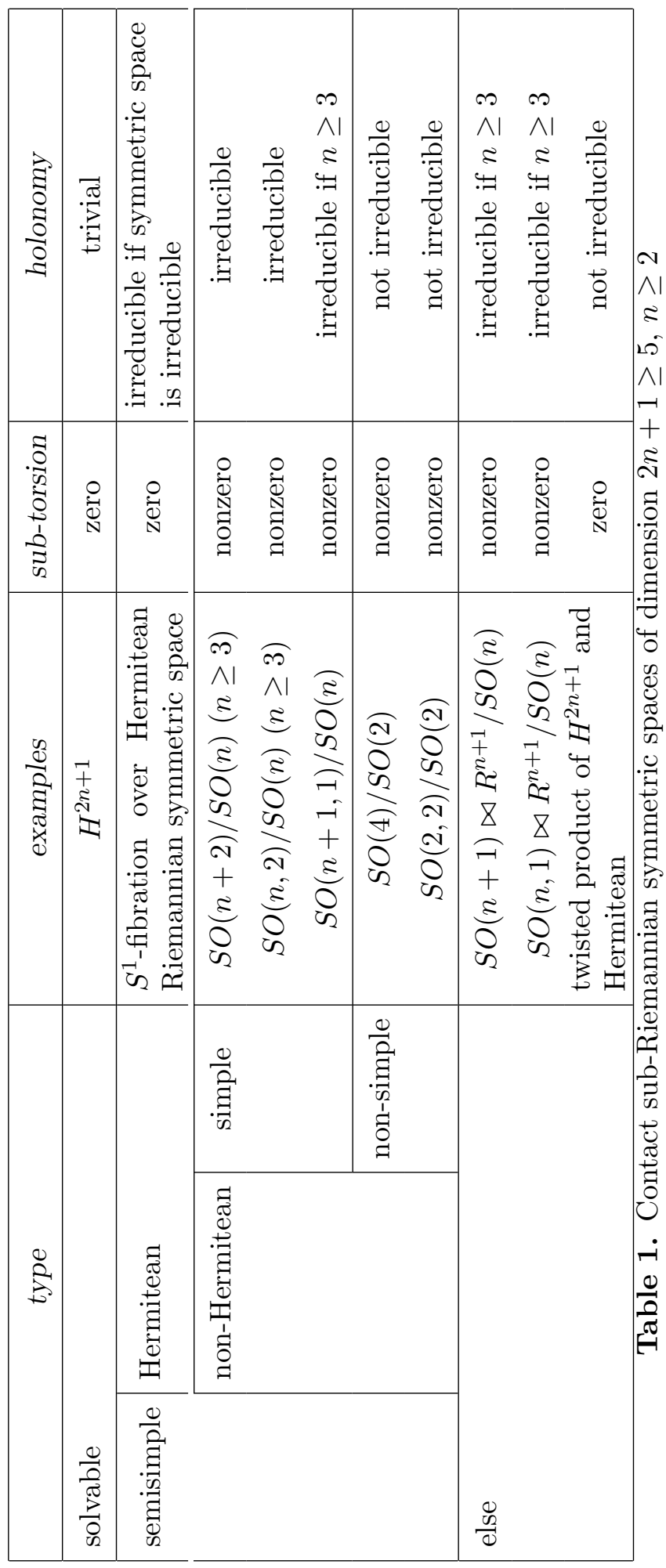




\section{References}

[1] P. Bieliavsky, Espaces symétriques symplectiques, Ph.D. thesis, Université Libre de Bruxelles, 1994-95.

[2] _ Four-dimensional symply-connected symplectic symmetric spaces, preprint, 1995.

[3] _ Symmetric coadjoint orbits of semisimple Lie groups, preprint, 1996.

[4] P. Bieliavsky, M. Cahen and S. Gutt, Deformation quantization and symmetric symplectic manifolds, in 'Modern Group Theoretical Methods in Physics', ser. 'Math. Studies', Kluwer Academic Publishers, 18 (1995), 63-75.

[5] _ A class of homogeneous symplectic manifolds, Preprint Université Libre de Bruxelles, 1995-96.

[6] A. Borel and J. de Siebenthal, Les sous-groupes fermés de rang maximum des groupes de Lie clos, Comment. Math. Helv., 23 (1949), 200-221.

[7] É. Cartan, Leçons sur la geométrie des espaces de Riemann, Gauthier-Villars, Paris, 1951.

[8] E. Falbel and C. Gorodski, On contact sub-Riemannian symmetric spaces, Ann. Sc. Éc. Norm. Sup., 28(4) (1995), 571-589.

[9] _ Sub-Riemannian homogeneous spaces in dimensions 3 and 4, Geom. Dedicata, 62(3) (1996), 227-252.

[10] E. Falbel, C. Gorodski and M. Rumin, Holonomy of sub-Riemannian manifolds, Intern. J. Math., 8(3) (1997), 317-344.

[11] E. Falbel, J.A. Verderesi and J.M. Veloso, The equivalence problem in sub-Riemannian geometry, Preprint IMEUSP, 1993.

[12] _ Constant curvature models in sub-Riemannian geometry, Mat. Contemp., Soc. Bras. Mat., 4 (1993), 119-125.

[13] F. Gantmacher, On the classification of real simple Lie groups, Math. Sbornik, 5 (1939), 101-144.

[14] M. Gromov, Carnot-Carathéodory spaces seen from within, IHES preprint M/94/6, 1994.

[15] S. Helgason, Differential Geometry, Lie Groups, and Symmetric Spaces, Academic Press, 1978.

[16] H. Jacobowitz, An Introduction to CR Structures, Amer. Math. Soc., 1990.

[17] S. Kobayashi and K. Nomizu, Foundations of Differential Geometry, Wiley Interscience Publishers, 1963-1969.

[18] S. Koh, Affine symmetric spaces, Trans. Amer. Math. Soc., 119 (1965), 291-301.

[19] I. Kupka, Géométrie sous-Riemanniene, Séminaire Bourbaki, Astérisque, 241 (1997).

[20] R. Montgomery, A survey of singular curves in sub-Riemannian geometry, J. Dyn. and Control Syst., 1(1) (1995), 49-90.

[21] G.D. Mostow, Self-adjoint groups, Ann. of Math., 62 (1955), 44-55.

[22] S. Murakami, Sur la classification des algèbres de Lie réeles et simples, Osaka J. Math., 2 (1965), 291-307.

[23] M. Rumin, Formes différentielles sur les variétés de contact, J. Diff. Geom., 39 (1994), 281-330. 
[24] R.S. Strichartz, Sub-Riemannian geometry, J. Diff. Geom., 24 (1986), 221-263.

[25] _ C Corrections to 'Sub-Riemannian geometry', J. Diff. Geom., 30 (1989), 595596.

[26] A.M. Vershik and V.Ya. Gershkovich, Non-holonomic Dynamical Systems, Geometry of Distributions and Variational Problems, Encyclopaedia of Mathematical Sciences series, 16 (1994), 1-81, Springer-Verlag; Russian original, 1987.

[27] S.M. Webster, Pseudo-Hermitian structures on a real hypersurface, J. Diff. Geom., 13 (1978), 25-41.

[28] J.A. Wolf, Spaces of Constant Curvature, Publish or Perish, Boston, 1974.

Received January 22, 1997 and revised February 12, 1998.

Pennsylvania State University

University Park State College, PA 16802

E-mail address: bieliap@math.psu.edu

Université de Paris VI

PARIS CEDEX 05

FRANCE

E-mail address: falbel@math.jussieu.fr

Universidade de SÃo PaUlo

05315-970 SÃo PaUlo, SP

BRAZIL

E-mail address: gorodski@ime.usp.br 